\title{
A Trusted Recommendation Method based on Social Circles
}

\author{
Chengjian Su, Genlang Chen, Guanghui Song \\ Ningbo Institute of Technology, Zhejiang University \\ Ningbo, Zhejiang, 315000, China
}

\begin{abstract}
The crisis of confidence has become increasingly rigorous, especially in recommendation domain. Deception and malicious attacks are found among the traditional collaborative filtering recommendation systems. Taking into account the credibility, the behaviour-based trust relationship, and the social circle-based trust relationship, this paper proposes a trusted recommendation method via social circle to enhance the credibility of recommendation. By using the social circles, which can significantly help to deal with the sparsity and trust problem of the traditional collaborative filtering, the method is aimed to solve two fundamental problems: accuracy and robustness. Empirical evaluation of the method on the dataset of last.fm demonstrates that the method can enhance the robustness without reducing the accuracy of the recommendation.
\end{abstract}

Keywords: collaborative filtering; credibility; trust model; social circle; recommendation system

\section{INTRODUCTION}

Online social networks generate millions of posts and allow us to cope with "information overload", which cause people have to reorganize our personal social networks [1]. Used in e-commerce, social media and other Internet applications, personalized recommendation technology has demonstrated the ability to help us to retrieve the preferences $[2,5,6]$. With further development of the recommendations, the crisis of confidence has become increasingly rigorous. Some serious security problems, such as recommended deception and malicious attacks, are found among the traditional collaborative filtering recommendation systems. Furthermore, the robustness of the recommendation method is becoming more and more important.

Some efforts have been made in the study of security and trust, researchers have focused on how to prevent attacks, improve the system's robustness and ensure the quality of recommendations. Montaner et al. propose a trust model, introduce the concept of trust factor [3]. They believe that the trust factor is dynamically changed along with the users' satisfaction. Schillo et al. present formalization and an algorithm for trust so that the model can autonomously deal with the deception [4].
They demonstrate with results that their approach helps to establish a model of trustworthiness.

Social circles are groups of socially interconnected people that can be used for content filtering, for privacy, and for sharing groups that others may wish to follow [1]. Practically all major social networks provide such functionality. Such as "circles" on Google+, Qzone, Facebook etc. People in Facebook, Google+, and Qzone may form "circles" based on common interests and attributes between themselves and the users whom they follow [1]. The common interests and attributes are just meeting the basic requirements of the recommendation. Just as in the real world, people usually ask friends or reliable people for advice on interesting items. And people only ask for advice to friends with similar tastes and interests who can be trusted. The solution we propose in this paper is a new recommendation method to improve the robustness and quality of recommendations based on social circles. Its main idea is to consider the other people which you can rely or not and build a trusted model based on social circle. Credibility is expressed through a trust value with which each people label its friends [3,11,17]. Therefore, the recommendation method we propose is based on a model of trust in the collaborative social circles.

By synthesizing the attributes of users, the behaviour of users and the relationship of social circles, this paper analyses the trusted features and networks of the recommendation system. From three aspects, including the user's credibility, the trust relationships between users based on recommended behaviours and the trust relationships of networks, this paper proposes a new trust model based on social circles, which enables to improve the robustness and quality of the collaborative filtering algorithm based on user behaviours. The rest of the paper is organized as follows. The next section provides a brief overview of trust models based on social circle and defines our problem domain. Section 3 describes the pre-processing approach adopted in this paper including the initialization of the trust factor and the feedback of the recommendation based on social circles. Section 4 describes our evaluation method, results, and discussion. The final section provides some concluding remarks. 


\section{NOTATION AND DEFINITIONS}

Traditional collaborative filtering systems can be cast as predicting the unknown ratings: given a user-item matrix of scores with many missing values, the goal of the traditional collaborative filtering method is to estimate the missing values on the basis of the similarity among profiles of different users [13]. However, when a similar user gives unsuccessful advice, there is no way to ignore it. Over and over again, the system will cause a descent in the performance [21]. Therefore, in this paper, trust networks, credibility and trust relationships are adopted to overcome it.

\subsection{Trust Model}

In real life, connections between people can form the social networks, which result in trust relationships being formed between people. Just as in the real world, the relationship between users can be used to form a trust network based on the social networks. As shown in Figure 1, the trust network is a directed graph, where each node represents a user, each edge represents the trust relationships between the users and the edge weight is the trust factor [11][15].

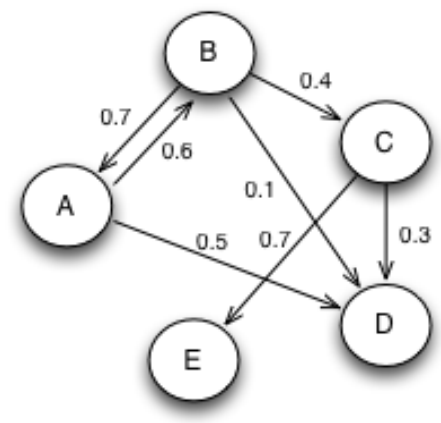

Fig. 1. A Trust Network

In real life, people tend to trust friends, individuals or organizations with integrity or credibility. Similarly, the trust model includes whether the recommended system is trusted itself, whether there exists a trust relationship between two users, and whether the users themselves trust each other. In default, the system is not considered to be suspect and it should be absolutely credible. Therefore, in studying the relationship of trust models, the method mainly considers two parts, the reliability and the credibility. The former is expressed through a trust value with which each people label his or her neighbours and the latter is a relatively fixed value, which is similar with one's reputation. The reliability between users is composed by the trust relationship based on users' behaviours, the trust relationship based on social circle and the undirected trust relationship.

\subsection{Credibility}

The concept of reliability and credibility is very similar, but there are some differences between them [4].
Just like the reputation, the credibility is an expectation, which is formed by the observation of a user's past behaviours or past information. In other words, the credibility emphasizes a comprehensive trustworthiness, which is given by a group. It is a relatively fixed value at one point of time. Conversely, the reliability emphasizes a subject reliance that is given by the individual. It is variable with different individuals. The credibility is defined as $\operatorname{Credit}\left(u_{i}\right)$ and the value means the credibility of user $u_{i}$.

Each user will have a degree of credibility and the value will be adjusted according to the following rules:

First, initialize the user's credibility. Give an initial value of the credibility according to the completion and authenticity of the user's profile. The more complete and authentic the profile, the higher the initial credibility is given.

Second, adjust the user's credibility. If one's recommendation is accepted by other users, the credibility will be enhanced. On the contrary, if one's recommendation is rejected by other users, the credibility will be reduced.

According to the rules, when building the trusted recommendation model, in addition to considering the similarity between $u_{i}$ and $u_{j}$, the method will also focus on the credibility of $u_{i}$. If one has a high degree of credibility, his recommended items may be more likely to be accepted by other users. Otherwise, they may be more likely to be rejected.

\subsection{Behaviour-based Trust Relationship}

In real life, when user $u_{i}$ recommends an item to user $u_{j}$, user $u_{j}$ eventually accepts and endorses the recommendation of user $u_{i}$. The next time and each following instant, user $u_{j}$ will believe user $u_{i}$ than ever. In fact, many recommendation systems have adopted the feedback mechanism now. In order to describe the trust relationship between the recommender and the nominee, the feedback is defined as:

$$
\operatorname{Ack}\left(u_{j}, u_{i}, a c t\right)=\left\{\begin{array}{l}
1, \text { accept the recommendation } \\
0, \\
-1, \text { reject the recommendation }
\end{array}\right.
$$

Similarly, the reliability between $u_{j}$ and $u_{i}$ is defined as $\operatorname{Trust}\left(u_{j}, u_{i}\right)$ and the value means the reliability of user $u_{j}$ to user $u_{i}$. 
Therefore, according to the definition of $\operatorname{Ask}\left(u_{j}, u_{i}, a c t\right)$, the feedback can be used to not only update the reliability of user $u_{j}$ to user $u_{i}$ but also adjust the credibility of the recommender $u_{i}$.

\subsection{Social circle-based Trust Relationship}

However, the above $\operatorname{Trust}\left(u_{j}, u_{i}\right)$ is only computed after user $u_{i}$ recommends an item to user $u_{j}$. In many cases, the trust matrix between user and user is very sparse. In addition, the cold start problem is very serious. Therefore, it is important to find a way to solve the problem of the initialization matrix.

The reliability $\operatorname{Trust}\left(u_{j}, u_{i}\right)$ represents the trust relationship between $u_{j}$ and $u_{i}$. As we all know, the social circle itself implies a trust relationship between users [11]. It is a viable option to initialize the trust matrix based on the social circle relationships.

In figure 1 , according to the degree of the node and the dependence between the users, a relationship can be built. The reliance of $u_{j}$ to $u_{i}$ can be presented as follows:

$$
\text { Friend }\left(u_{j}, u_{i}\right)=\left\{\begin{array}{c}
\frac{1}{k\left(u_{j}\right)}, \text { if } u_{j} \text { is a non isolated point } \\
0, \text { if } u_{j} \text { is a isolated point }
\end{array}\right.
$$

Where $k\left(u_{j}\right)$ is the degree of $u_{j}$. For example, the degree of $u_{a}$ is 3 , the reliance of $u_{a}$ to $u_{b}, u_{c}$ and $u_{d}$ is $\frac{1}{3}$.

In this paper, the idea is utilizing the reliability between users of social circle $\operatorname{Friend}\left(u_{j}, u_{i}\right)$ to initialize the $\operatorname{Trust}\left(u_{j}, u_{i}\right)$. It can be initialized as follows:

$$
\operatorname{Trust}\left(u_{j}, u_{i}\right)=\operatorname{Trust}\left(u_{j}, u_{i}\right)+\alpha \cdot \operatorname{Friend}\left(u_{j}, u_{i}\right)
$$

Where $\alpha$ is a variable factor. It can be adjusted according to the impact of the social circle.

\subsection{Indirect Trust Relationship}

In social circle, the trust has a transitive, but it is not absolute. For example, if $u_{b}$ is trusted by $u_{a}$ and $u_{c}$ is trusted by $u_{b}$, it may be inferred that there is a certain indirect trust relationship exists between $u_{a}$ and $u_{c}$. Although the indirect trust relationship may not be fully established, to some extent, it can be used to solve the sparsely problem of the trust matrix.

\section{TRUSTED MODEL BASED ON SOCIAL CIRCLE}

Traditional collaborative filtering method estimates the missing values on the basis of the similarity among users' profiles, items' features and users' behaviours [13]. In this paper, the method adopts the value of trust to determine the nearest neighbours.

\subsection{Estimate Trusted Relationships}

In this method, the value of trust can be decided by the credibility of $u_{i}$ and the feedback of $u_{j}$ to $u_{i}$. Commonly, if the credibility of $u_{i}$ is much higher, it can be inferred that $u_{i}$ is more trustworthy. The value of trust is computed as follows:

$$
\operatorname{Trust}\left(u_{j}, u_{i}\right)=\frac{\sum_{u_{j k} \in u_{j} \cap u_{i k} \in u_{i}} \operatorname{Ack}\left(u_{j k}, u_{i k}, a c t\right)}{\left|\operatorname{Ack}\left(u_{j}, u_{i}, a c t\right)\right|}
$$

Where $\operatorname{Ack}\left(u_{j}, u_{i}, a c t\right)$ means the total number that $u_{i}$ once recommended to $u_{j}, 0 \leq \alpha \leq 1$ is a variable factor. When $\alpha$ equals to 0 , it means the trust is completely decided by the credibility of $u_{i}$. On the contrary, when $\alpha$ equals to 1 , it means the trust is completely decided by the feedback between $u_{j}$ and $u_{i}$. In Equation (4), if $A c k\left(u_{j}, u_{i}, a c t\right)$ is negative, it means $u_{j}$ rejects the recommendation of $u_{i}$ and the credibility of $u_{i}$ will be punished by this act. According to the feedback, the credibility will be corrected as follows:

$\operatorname{Credit}\left(u_{i}\right)=\operatorname{Credit}\left(u_{i}\right)+\frac{\operatorname{Trust}\left(u_{j}, u_{i}\right)}{\operatorname{Credit}\left(u_{i}\right)} \cdot \operatorname{Credit}\left(u_{j}\right)$

In order to prevent the malicious fraud and attacks to affect the credibility of $u_{i}$, in formula 5 , it includes the credibility of $u_{j}$ to reduce the influence of improper feedback.

\subsection{Build Trusted Model}

In this model, it mainly considers the credibility, the 
behaviour-based trust relationship and the social circlebased trust relationship. The indirect trust relationship is not included in this model. The model is built as follows:

Step 1: initialize the user's credibility vector $C$ redit . According to the completion and authenticity of the user's profile, the credibility of users is initialized to four levels, corresponding to $\{0,0.05,0.1$, and 0.15$\}$ based on the observation of the previous experiments and empirical values.

Step 2: initialize the trust matrix Trust. It will be initialized on the basis of social circle relationship according to the Equation (2) and Equation (3).

Step 3: build the feedback mechanism between users and save the result to the feedback matrix Ack.

Step 4: update the credibility and trust matrix. According to the feedback of step 3 and the Equation (5), the Credit and Trust will be updated again and again.

Finally, the latest credibility will be saved in credibility vector Credit and the latest trust will be saved in trust matrix Trust .

\subsection{Prediction and Recommendation}

It is similar to the traditional collaborative filtering method. Correlation similarity is used to compute the similarity between user and user. Using the behaviours that have been acted by both users and using the trust and credibility of users compute the similarity between user and user. The similarity is computed as follows:

$$
\begin{gathered}
\operatorname{TSim}\left(u_{j}, u_{i}\right)=\alpha \cdot \operatorname{Sim}\left(u_{j}, u_{i}\right)+\beta \cdot \operatorname{Trust}\left(u_{j}, u_{i}\right) \\
+\gamma \cdot \operatorname{Credit}\left(u_{i}\right) \\
\alpha+\beta+\gamma=1
\end{gathered}
$$

where $\operatorname{Sim}\left(u_{j}, u_{i}\right)$ means the similarity based on the behaviours. It can be computed by using Pearson correlation [17], which is most popular and widely used.

By setting different combinations of $\alpha, \beta$ and $\gamma$, this method can find the top-k neighbours that are the most similar, the most trusted and the most reputable to user $u_{j}$.

Based on the similarity, it can get the most similar knearest neighbours $U(u, k)=\left\{u_{1}, u_{2}, u_{3}, \ldots u_{k}\right\}$. Then according to the nearest neighbours $U(u, k)$, the final step of the method is to predict the rating of the active user on all uncollected services and generate the top-n recommended lists [18]. The predictions can be computed as follows:

$$
r(u, s)=\sum_{v \in U(u, k) \cap N(s)} T \operatorname{sim}(u, v) * r(v, s)
$$

where $N(s)$ is the user set that has acted on service
$S$ and $r(v, s)$ is the rating of user $v$ on service $S$.

Finally, according the value of predictions, the top-n services of highest value are selected as the recommended lists.

\section{EXPERIMENTAL EVALUATION}

In order to evaluate the performance of the proposed method, we applied the method to real data sets of last.fm, and compared the accuracy and robustness of different methods. In this section we present a brief discussion of our experimental data set, evaluation metric, the experimental results and discussion.

\subsection{Data Sets}

In this paper, we used the information of last.fm, which is the world's largest social music service. This dataset contains 1892 users, 17632 artists, 12717 twoway friendships, 92843 user behaviours, 11946 tags and 186479 tag behaviours. Taking into account the data set does not contain a clear degree of credibility, trust index and feedback, in the experiment, this paper made the following assumptions:

First, set the initial value of credibility and trust index on the basis of the friend relationship among the dataset. The more the number of friends of one owned, the higher is the degree of his credibility.

Second, consider the behaviours of listening music as a measure of similarity between users.

Third, according to the time series of tag behaviours, the credibility and trust index are adjusted between users. In the experiment, assuming the number of common tags between two users' increases with time sequence, the trust index between the two users will also be enhanced.

At last, in order to verify the accuracy, we divided the database into $90 \%$ training set and $10 \%$ test set.

\subsection{Evaluation Metrics}

In order to measure the performance, we focused on two evaluation metrics corresponding to prediction accuracy and robustness. The Root Mean Square Error (RMSE) [13] is widely used as a statistical accuracy metric to evaluate the precision. The robustness is estimated in terms of the error rate of the recommendation.

For each ratings-prediction pair $\left\langle p_{i}, q_{i}\right\rangle$, the formula of RMSE is computed as follows:

$$
R M S E=\frac{\sqrt{\sum_{i=1}^{N}\left(p_{i}-q_{i}\right)^{2}}}{N}
$$

Equation (8) means the lower the RMSE, the more accurately the recommendation engine predicts user ratings. 
We define the error rate as the number of occurrences that the noisy items are recommended by the system. The comparison results with User CF method, which is the typical collaborative filtering method based on users, will be provided through the experiments.

\subsection{Results and Discussion}

Fig. 2 shows the comparison of the RMSE between collaborative filtering based on user behaviours (User CF) and trusted recommendation (Trust CF) method. As shown in Fig.2, the prediction quality of Trust CF is obviously better than User CF. It also verified the reallife situations that people are more inclined to trust their own friends' recommendations. Therefore, in the field of social media recommendation, the trust relationship between friends has great influence on the recommendation.

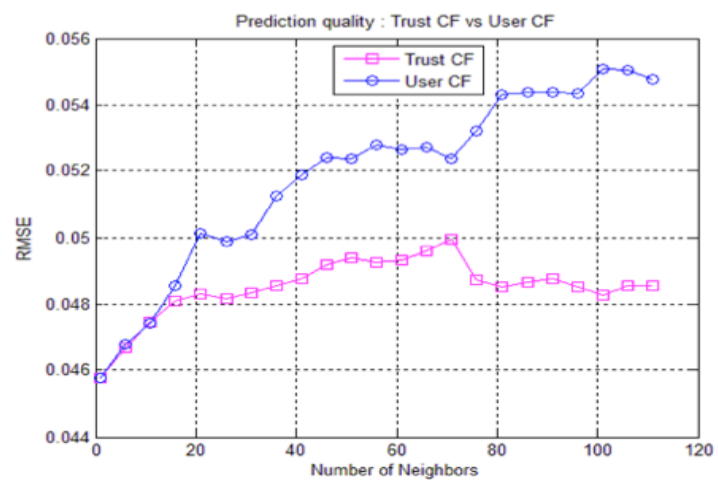

Fig.2. Comparison of the RMSE

According to the Equation (6), the weight of trust index can be adjusted appropriately, thus the trust recommendation model can be changed accordingly. The study has showed that, to some extent, the weight of the trust can affect the accuracy of the recommendation. As shown in figure 3, within a certain range, the higher the percentage of the trust, the better is the accuracy.

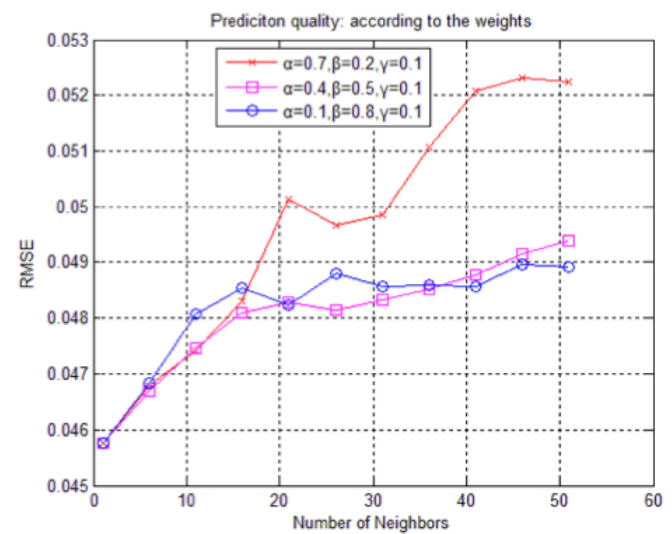

Fig.3. Comparison of the RMSE for different weights

In order to verify the robustness of the method, especially when it is faced the prop attacks of the disguised-users. In the experiment, we compared the error rate of top- $\mathrm{N}$ to verify the robustness of the method. The specific methods are as follows:

First, the dataset is divided into two groups. One is the normal data and the other is abnormal data, which is including the attack data. As shown in table 1, those items, which are labelled from 2001 to 2009, have been attacked by the positive evaluation.

Second, get the top-10 lists of Trust CF and User CF separately by using normal data and abnormal data.

Third, compare the result of the two different methods.

From table 1, it can be observed that, for Trust CF method, the rate of 2001 to 2009 appeared in top-10 is increased from $30 \%$ to $50 \%$ after adding the noisy data. But, for User CF method, the rate is increased from 30\% to $80 \%$. Therefore, in this experiment, the Trust CF method shows better adaptability and robustness to the noisy data than User CF. It is due to the reason that Trust CF method not only concerned with users' reviews, but also concerned about the degree of trust between users.

TABLE 1. Comparison of the Robustness for Trust CF and User CF

\begin{tabular}{|l|l|l|l|}
\hline \multicolumn{2}{|c|}{ Trust CF(Top-10 item id) } & \multicolumn{2}{c|}{ User CF(Top-10 item id) } \\
\hline Normal Data & $\begin{array}{c}\text { Abnormal } \\
\text { Data }\end{array}$ & Normal Data & $\begin{array}{c}\text { Abnormal } \\
\text { Data }\end{array}$ \\
\hline 2008 & 2008 & 2008 & 2008 \\
\hline 1005 & 1005 & 2004 & 2004 \\
\hline 1006 & 2002 & 1006 & 2001 \\
\hline 2002 & 2000 & 2009 & 2002 \\
\hline 2000 & 1001 & 1002 & 2009 \\
\hline 1001 & 2003 & 2000 & 2007 \\
\hline 72 & 2009 & 1003 & 2003 \\
\hline 1003 & 2004 & 1005 & 2005 \\
\hline 2003 & 72 & 1001 & 2000 \\
\hline 1005 & 1003 & 72 & 2006 \\
\hline
\end{tabular}

\section{CONCLUSION AND FUTURE WORK}

Recently, the trust and security issues have been a new field within the recommendation domain. In this paper we have proposed a new trust-based recommendation method based on the following two points. First, the good credibility of a man is more worthy of trust. Second, the more help to others, the more worthy of trust. By using the credibility, the behaviour-based trust relationship and the social circlebased trust relationship, an updated collaborative filtering method is proposed and it is aimed to two fundamental problems: accuracy and robustness. Experimental results show that our proposed method can enhance the robustness as possible as without reducing the accuracy.

For future work, we will enlarge the dataset to confirm the real-time response speed, robustness and accuracy of the algorithm. Besides, it is also interesting to see the performance of the approach when it facing the big data and in other domains. Furthermore, we wish to find better algorithms to speed up by using GPU that can be used to improve the real-time response speed. 


\section{CONFLICT OF INTEREST}

The authors confirm that this article content has no conflicts of interest.

\section{ACKNOWLEDGMENT}

This work is partially supported by Zhejiang Provincial Natural Science Foundation, China (No. LY15F020035) and Ningbo Natural Science Foundation, China (No. 2015A610125).

We also acknowledge the Last.fm to share the relevant data.

\section{REFERENCES}

[1] Julian Mcauley, Jure Leskovec, "Discovering social circles in ego networks," ACM Transactions on Knowledge Discovery from Data, Vol.8, No. 1, Article 4, 2014.

[2] Soo Ling Lim, Finkelstein, A. StakeRare, "Using Social Networks and Collaborative Filtering for Large-Scale Requirements Elicitation”, Software Engineering, IEEE Transactions on, 38(3): pp. 707 735, 2012.

[3] M. Montaner, B. López and J. Lluís de la Rosa, “Opinion-Based Filtering through Trust”, Lecture Notes in Computer Science, 2446(2002), pp. 127 144, 2002.

[4] Michael Schillo, Petra Funk, Michael Rovatsos, "Using Trust for Detecting Deceitful Agents in Artificial Societies”, Applied Artificial Intelligence, vol. 14, pp. 825-848, 2000.

[5] Massa P., Bhattacharjee B., "Using Trust in Recommender Systems: an experimental analysis”, In proceedings of Second International Conference on Trust Management, pp. 221 235, 2004.

[6] Jie Liu, Mingsheng Shang, Duanbing Chen, "Personal Recommendation Based on Weighted Bipartite Networks", 2009 Sixth International Conference on Fuzzy Systems and Knowledge Discovery, pp. 134 137, 2009.

[7] Junhai Luo, Xue Liu, Yi Zhang, Danxia Ye, Zhong Xu, "Fuzzy Trust Recommendation Based on Collaborative Filtering for Mobile Ad-hoc Networks”, in Proceedings of the 33rd Annual IEEE Conference on Local Computer Networks, Montreal, QC, Canada, pp. 305-311, 2008.

[8] R. Badonnel, R. State, O. Festor, A. Schaff, “A framework for optimizing end-to-end connectivity degree in mobile ad-hoc networks”, J. Netw. Syst. Manage., vol. 13, no. 4, pp. 479-497, 2005.

[9] Y. L. Sun, Y. Yang, "Trust establishment in distributed networks: Analysis and modeling”, Communications, 2007. ICC '07. IEEE International Conference on, pp. 1266-1273, 24 28, 2007.

[10] Fuzhi Zhang, Huan Wang, Huawei Yi, "An Adaptive Recommendation Method Based on Small-World Implicit Trust Network”, Journal of Computers, vol. 9, no. 3, pp. 618-625, 2014.

[11] J. Seo, S. Choi, S. Han, "The Method of Trust and Reputation Systems Based on Link Prediction and Clustering”, Trust Management VII, Springer Berlin Heidelberg, pp. 223-230, 2013.

[12] W. Yuan, D. Guan, Y. K. Lee, et al., "Improved Trust-aware Recommender System Using Small-worldness of Trust Networks”, Knowledge-Based Systems, vol. 23, no. 3, pp. 232238, 2010.
[13] Genlang Chen, Xiaogang Jin, Jiangang Yang, “A Collaborative Filtering Algorithm via Clustering in Mobile Service", International Journal of Advancements in Computing Technology, vol. 4, no. 13, pp. 415-421, 2012.

[14] Yu Wang, Lin Gao, “An Edge-based Clustering Algorithm to Detect Social Circles in Ego Networks”, Journal of Computers, vol. 8, no. 10, pp. 2575-2582, 2013.

[15] M. Girvan and M. Newman, "Community Structure in Social and Biological Networks”, Proc Natl Acad Sci, vol.99, no. 12, pp. 7821-7826, 2002.

[16] Khalid EI-Arini, Gaurav Veda, Dafna Shahaf, Carlos Guestrin, "Turning Down the Noise in the Blogosphere", Proceedings of the 15th ACM SIGKDD international conference on Knowledge discovery and data mining, pp. 289-298, 2009.

[17] Manh Cuong Pham, Yiwei Cao, Ralf Klamma, Matthias Jarke, " A Clustering Approach for Collaborative Filtering Recommendation Using Social Network Analysis”, Journal of Universal Computer Science, vol. 17, no. 4, pp. 583-604, 2011.

[18] Ma, H., Lyu, M. R., King I., "Learning to Recommend with Trust and Distrust Relationships", In Proceeding(s) of the third ACM conference on Recommender systems, pp.189-196, 2009.

[19] Mohammad Julashokri, Mohammad Fathian, Mohammad Reza Gholamian, Ahmad Mehrbod, "Improving Recommender System's Efficiency Using Time Context and Group Preferences”, AISS: Advances in Information Sciences and Services Sciences, vol. 3, no. 4, pp. 162-168, 2011.

[20] Heng-Li Yang, Hsiao-Fang Yang, "Recommendation Mechanism Based on Multi-attribute Utility Theory", JDCTA: International Journal of Digital Content Technology and its Applications, vol 5, no. 3, pp. 373-382, 2011.

[21] Fuzhi Zhang, Shuangxia Sun, “A Robust Collaborative Recommendation Algorithm Based on Least Median Squuares Estimator”, Journal of Computers, vol. 9, no. 2, pp. 308-314, 2014.

[22] Songjie Gong, "A Collaborative Filtering Recommendation Based On Trust Network and Trust Factor", Journal of Convergence Information Technology, vol. 8, pp. 1111-1118, 2013. 